\title{
ON THE SPECTRUM OF A DISTRIBUTION FUNCTION AND ON UNIQUE FACTORIZATION
}

\author{
T. PHAM-GIA \\ Departement de Physique-Mathematiques \\ Universite de Moncton \\ Moncton, N.-B., E1A 3E9 CANADA \\ (Received November 13, 1980 and in revised form June 30, 1982)
}

\begin{abstract}
The spectrum of a distribution function is related to the quasi-analyticity of a class of functions $\left\{\mathrm{C} \mathrm{M}_{(j)}\right\}$, where $M_{(j)}$ is a multisequence of positive numbers. For a regular multisequence, a result on the uniqueness of characteristic function decomposition is given.
\end{abstract}

KEY WORDS AND PHRASES. Distribution, characteristic function, spectrum, quasianalytic, regular, factorization.

1980 MATHEMATICS SUBJECT CLASSIFICATION CODES. Primary 26A93, 60005.

\section{INTRODUCTION.}

A general principle formulated by $\mathrm{N}$. Wiener, that a function and its Fourier transform cannot both be very small at infinity, has been the origin of important but quite different results in the fields of Complex Analysis and Probability Theory. In this note, we give some results connecting these two fields and an example to illustrate this connection.

Two fundamental theorems on the above principle are recalled below. They both rely on the classical Paley-Wiener Theorems. Theorem 1.1 by Ingham and Levinson [1] has been used by various authors to establish results in Probability Theory, concerning the spectrum of a distribution, unique factorization of characteristic functions, etc. (see e.g. [2], [3]). Theorem 1.2 by S. Mandelbrojt [4] has seen many applications in Analysis, especially in the theory of quasi-analytic and non-quasi-analytic functions (see e.g. [4], [5], [6]). 
THEOREM 1.1. Let $\mathrm{G}(\mathrm{u}) \in \mathrm{L}(-\infty, \infty)$ and suppose that

$$
F(x)=\frac{1}{2 \pi} \int_{-\infty}^{\infty} G(u)^{-i u x} d u
$$

Let $G(u)=O\left(e^{-\theta(u)}\right)$ where $\theta(u)$ is a non-decreasing positive function such that $\int_{1}^{\infty} \frac{\theta(u)}{u^{2}} d u=\infty$

Then $F(x)$ cannot be equivalent to zero in any interval unless it is equivalent to zero over $(-\infty, \infty)$.

THEOREM 1.2. If $\mathrm{C}(\mathrm{u})$ is a non-decreasing function on $[0, \infty)$ with $\int_{1}^{\infty} \frac{\mathrm{C}(\mathrm{u})}{\mathrm{u}^{2}} \mathrm{du}<\infty$, then $\mathbf{V} \delta, \exists$ an entire function $F(z)$ not identically zero such that

$$
|F(z)| \leq e^{\delta|y|-C(|z|)}
$$

A number of definitions and other results to be used later are recalled below.

A point $x$ is called a point of the spectrum of a probability distribution $F$ if $V \delta>0, F(x+\delta)>F(x-\delta)$. The characteristic function $f$ of $F$ is defined by $f(t)=\int_{-\infty}^{\infty} e^{i t x} d F(x)$ and the following theorem can be found in [2], under a slightly different form.

THEOREM 1.3. Let $\theta(t)$ be a non-negative non-decreasing function for $t>0$ and let $f(t)$ be the characteristic function of a distribution $F$ such that $f(t)=\mathbf{O}\left(e^{-\theta(|t|)}\right)$ for large $|t|$. Then $F$ has $(-\infty, \infty)$ as spectrum if and only if the integral $\int_{1}^{\infty} \frac{\theta(t)}{t^{2}} d t$ diverges.

The proof of the above result is essentially based on Theorem 1.1 and various properties of the Rademacher Series (see [2]).

2. IRAIN RESULTS.

Let $\left\{M_{(j)}\right\}$ be a multisequence of positive numbers where $(j)=\left(j_{1}, \ldots, j_{m}\right), j_{k}$ being an integer $\geq 0,1 \leq k \leq \mathrm{m}$. We set $|(j)|=\sum_{k=1}^{m} j_{k}$ and denote by $C\{M(j)\}$ the class of $\mathrm{C}^{\infty}$ complex-valued functions defined on $\mathrm{R}^{\mathrm{m}}$ such that

$$
\left.|| \frac{\partial|(j)|}{\partial x_{1}{ }^{j_{1}}{ }_{x_{m}}^{j_{m}}} f\right|_{\infty} \leq \alpha_{f} B_{f}|(j)|_{(j)}, \forall(j)
$$

where $\alpha_{f}$ and $B_{f}$ are positive constants which depend only on $f$. Following Lelong [7], we define the class $\mathrm{C}\left\{\mathrm{M}_{(j)}\right\}$ to be quasi-analytic if it does not contain functions with 
compact support. Various properties of these classes have been obtained (see [7],[8], and [9]) and differ from the classical one-dimensional case.

THEOREM 2.1. Let $F$ be a probability distribution function with characteristic function $f$ and $\left\{M_{(j)}\right\}$ a multisequence of positive numbers such that .

$\sup _{(j)}\left\{|| t|(j)| f(t)||_{\infty} / M_{(j)}\right\}<\infty$.

Then $F$ has $(-\infty, \infty)$ as spectrum if and only if $C\left\{M_{(j)}\right\}$ is quasi-analytic.

PROOF. We begin by extending the ideas of Valiron points and half-planes to several dimensions.

Let $\left\{M_{(j)}\right\}$ be a multisequence of positive numbers where $(j)=\left(j_{1}, \ldots, j_{m}\right), j_{k}$ being an integer $\geq 0$. We set $\mu_{p}=\underset{|(j)|=p}{\inf \left\{M_{(j)}\right\}}$ and suppose that $\left\{\mu_{p}\right\}$ grows more rapidly than any function of the form $r^{\mathrm{P}}, \mathrm{r}>0$.

In $\mathrm{R}^{\mathrm{m}+1}$, let's consider the points with co-ordinates $x_{1}=j_{1}, \ldots, x_{m}=j_{m}$ and $y=-\log M_{(j)}$, called the Valiron points of the sequence $\left\{M_{(j)}\right\}$, in the lower halfspace of $R^{m+1}$. Since $\frac{r^{p}}{\mu_{p}} \rightarrow 0, r>0$ and $a>0$, there exist at most a finite number of indices $\mathrm{p}$ such that $\mathrm{p} \log \mathrm{r}-\log \mu_{\mathrm{p}}>\mathrm{a}$. A fortiori, $\mathbf{V}(\mathrm{r})=\left(\mathrm{r}_{1}, \ldots, \mathrm{r}_{\mathrm{m}}\right)$ and a $>0$; there are at most a finite number of Valiron points such that

$$
\sum_{k=1}^{m} j_{k} \log r_{k}-\log M_{(j)} \geq a \text {. }
$$

Now fix (r) and fix $a>0$. Let's consider the hyperplane in $\mathrm{R}^{\mathrm{m}+1}$ determined by the equation:

$$
y=-\sum_{k=1}^{m} x_{k} \log r_{k}+a
$$

It intersects the $y$-axis at the point with ordinate a and the $x_{\ell}$-axis at the point $\frac{a}{\log r_{\ell}}$ with $1 \leq \ell \leq \mathrm{m}$. By $(2.1), \exists b=b\left(r_{1}, \ldots, r_{m}\right)$ such that no Valiron point is above $y=-\sum x_{k} \log r_{k}+b\left(r_{1}, \ldots, r_{m}\right)$ and, at the same $t i m e$, at least one Valiron point is on the hyperplane itself. Hence, for Valiron points, we have

$$
-\sum_{k=1}^{m} j_{k} \log r_{k}+b\left(r_{1}, \ldots, r_{m}\right) \geq-\log M_{(j)}
$$

or

$$
b\left(r_{1}, \ldots, r_{m}\right) \geq \sup _{(j)}\left\{\sum_{k=1}^{m} j_{k} \log r_{k}-\log M_{(j)}\right\} ;
$$


in fact

$$
\begin{aligned}
b\left(r_{1}, \ldots, r_{m}\right) & =\sup _{(j)}\left\{\log \left(\frac{r_{1}^{j_{1}}, \ldots, r_{m}^{j_{m}}}{M_{(j)}}\right)\right\} \\
& =\log T\left(r_{1}, \ldots, r_{m}\right)
\end{aligned}
$$

where

$$
T\left(r_{1}, \ldots, r_{m}\right)=\sup _{(j)}\left\{\frac{r_{1}^{j_{1}}, \ldots, r_{m}^{j_{m}}}{M_{(j)}}\right\}
$$

For $(r)=\left(r_{1}, \ldots, r_{m}\right)$ given, the hyperplane $H((r))$ with equation $y=-\sum_{k=1}^{m} x_{k} \log r_{k}+b\left(r_{1}, \ldots, r_{m}\right)$ is called the Valiron hyperplane of the sequence $\left\{M_{(j)}\right\}$ at the point $(r) \in R^{m}$.

We set $r_{1}=r_{2}=\ldots=r_{m}=r$ and consider the Valiron hyperplanes defined on the main diagonal of $R_{m}$, whose equations become $y=-\log r \sum_{k=1}^{m} x_{k}+b(r)$. Here $b(r)=\log \lambda(r)$ where $\lambda(r)=T(r, r, \ldots, r)$. We can see from the above arguments that $b(r)$ is a positive non-decreasing function of $r>0$.

Suppose now that $C\left\{M_{(j)}\right\}$ is quasi-analytic. By [8] we know that $\int_{1}^{\infty} \frac{b(r)}{r^{2}} d r$ diverges.

Let

Then $\sup _{(j)}\left\{|| t|(j)| f(t)||_{\infty} / M(j)\right\}=\gamma<\infty$.

$$
|f(t)| \leq\left.\gamma \frac{M}{|t|(j)}\right|^{\mid(j)} V^{(j)}
$$

and hence

$$
|f(t)|=0(1 / \gamma(|t|)) \text { as } t>\infty \text {. }
$$

Using Theorem 1.3, F then has $(-\infty, \infty)$ as spectrum. Conversely, suppose that $\mathrm{F}$ has $(-\infty, \infty)$ as spectrum. Since $|f(t)|=0\left(e^{-b(|t|)}\right) t \rightarrow \infty$, the integral $\int_{1}^{\infty} \frac{b(t)}{t^{2}} d t$ diverges again by [8], this is a sufficient condition for the class $\mathrm{C}\{\mathrm{M}(j)\}$ to be quasianalytic.

For one dimension, we have the following example and corollary.

EXAMPLE. If we associate to the Cauchy distribution (with density $p(x)=\frac{\theta}{\pi\left[\theta^{2}+(x-\mu)^{2}\right]},-\infty<x<\infty, \theta, \mu$ real,$\left.\theta>0\right)$ the sequence $M_{j}=\left(\frac{j}{\theta e^{\theta}}\right)^{j}$, we can easily verify that the above theorem is satisfied. 
COROLLARY. Let $\left\{M_{j}\right\}_{j=0}^{\infty}$ be a sequence of positive numbers such that $M_{0}=1$, $M_{n}^{2} \leq M_{n-1} M_{n+1}$ with $n=1,2,3, \ldots$, and $F$ is a distribution fuction with characteristic function $f$ such that $\sup _{j \geq 0}\left\{|| t^{j} f(t)||_{\infty} / M_{j}\right\}<\infty$. Then $F$ has $(-\infty, \infty)$ as spectrum if and only if $\sum_{n=1}^{\infty} \frac{M_{n-1}}{M_{n}}=\infty$.

PROOF. By the classical Denjoy-Carleman Theorem (see [9]), if $\mathrm{M}_{j}$ is logarithmically convex, the divergence of the integral $\int_{1}^{\infty} \frac{b(t)}{t^{2}} d t$ is equivalent to the divergence of the series $\sum_{n=1}^{\infty} \frac{M_{n-1}}{M_{n}}$.

3. UNIQUE FACTORIZATION.

An interesting property of distribution functions is that the cancellation law does not hold in general, i.e. $F_{1} * F_{2}=F_{1} * F_{3}$ does not imply $F_{2}=F_{3}$. Gnedenko [10] has given examples of characteristic function $f$ such that $f(t)=f_{1}(t) f_{2}(t)=$ $f_{1}(t) f_{3}(t)$ with $f_{2}(t) \neq f_{3}(t)$.

For a distribution function $F$, we define $F^{*}(x)=F(-x), \forall x$ and, for $a>0$, $\left(\Delta_{a} F\right)(x)=F(x+a)-F(x-a)$

We now consider a more specific multisequence called regular. For $\left\{M_{(j)}\right\}, 1$ et's associate the marginal multisequence $\left\{M_{(j)}^{*}\right\}$ defined by

$$
\begin{array}{r}
M_{(j)}^{*}=M_{(j) \mid, 0, \ldots,}{ }_{0,|(j)|, 0, \ldots, 0}^{M_{0}} \\
\ldots M_{0}, 0, \ldots,|(j)|, \forall(j)
\end{array}
$$

DEFINITION. A multisequence $\left\{M_{(j)}\right\}$ is regular if:

1) $M_{(j)} \geq M_{(j)}^{*} \forall(j)$, with $\sup _{(j)}\left\{\left(\frac{M(j)}{M_{(j)}^{*}}\right)\right\}<\infty$.

2) $\left\{\mathrm{C} \mathrm{M}_{(\mathrm{j})}^{*}\right\}$ is quasi-analytic with each marginal sequence $\left\{\mathrm{M}_{0}, \ldots, 0, \ell, 0, \ldots, 0\right\}$ of the form $\mathrm{M}_{0}, \ldots, \ell, \ldots, 0=(\ell !)^{\alpha_{k}}[v(\ell)]$ where $\sum_{k=1}^{m} \alpha_{k}=1$ and $v(x)$ is a continuous $1 y$ differentiable function (depending on the marginal sequence) with $V(0)=1, V^{\prime}(x) \geq 0$ and $\frac{x \nu^{\prime}(x)}{V(x)}=0(1)$ as $x \rightarrow \infty$.

REMARK. The class of regular multisequence, which is obviously not empty, was introduced first by Hirschman [11] for one dimension, to generalize the m-th logarithmic sequence. 
THEOREM. Let $\mathrm{C}\left\{\mathrm{M}_{(\mathrm{j})}\right\}$ be a regular class of functions and $\mathrm{f}$ the characteristic function of $F$. Then there exists a continuous non-decreasing function $\psi(t)$ for $t>0$ such that:

1) If $f(t)=0\left(e^{-|t| / \Psi(|t|)}\right)$, then $F$ has $(-\infty, \infty)$ as spectrum

2) If $\left(\Delta_{a} F^{*}\right)(u)=0\left(e^{-|t| / \Psi(|t|)}\right)$ for some $a>0$ and $f$ admits a decomposition $f(t)=f_{1}(t) f_{2}(t)$, then $f_{2}$ is uniquely determined by $f$ and $f_{1}$.

PROOF. We first show that, for a regular multisequence $\left\{M_{(j)}\right\}$, the function $b *(r)$ associated with the marginal multisequence $\left\{M_{(j)}\right\}$ is such that $b *(r) \sim \frac{r}{\psi(r)}$ as $r \rightarrow \infty$ where $\psi(r)$ is a continuous, non-decreasing function with $\psi(0)>0$.

We have

$$
\lambda *(r)=\sup _{p \geq 0}\left\{\frac{r^{p}}{M^{\star}}\right\}
$$

with

$$
\begin{array}{r}
\left.\mu_{p}^{*}=\inf _{|(j)|=p}\left\{\mathrm{H}_{(j)}^{*}\right\}=(p !)^{\alpha}{ }^{\alpha} \nu_{1}(p)\right]^{p} \ldots(p !)^{\alpha_{m}} \\
{\left[\nu_{m}(p)\right]^{p}=p ![\nu(p)]^{p} \text { where } \nu=\prod_{k=1}^{m} \nu_{k} \cdot}
\end{array}
$$

We also have $V(0)=1, V^{\prime}(x) \geq 0$, and $\frac{x \nu^{\prime}(x)}{V(x)}=0(1)$ as $x \quad \infty$.

Following Hirschman [11], write

$$
b *(r)=\max _{p>0}\{p \log r-p !-p \log v(p)\}
$$

and let $p^{*}(r)$ be the integer for which the maximum is attained. If we now treat $p$ as a continuous riable in $G(p)=p \log r-p !-p \log V(p)$, the maximum would be attained as $\mathrm{s}$ ), the solution of $\frac{d G(p)}{d p}=0$. Using Stirling's formula $\mathrm{p} ! \sim \sqrt{2 \mathrm{rp}} \mathrm{p}^{\mathrm{p}} \mathrm{e}^{-\mathrm{p}}$, we have $\frac{d}{d p}(\log \Gamma(\mathrm{p}+1))=\log \mathrm{p}+\bullet(1)$ and, therefore, $\frac{d G(p)}{d p}=\log r-\log p-\log v(p)+o(1)$ by the properties of $v$. Hence,

$$
\log r=\log s(r)-\log v(s(r))+\bullet(1)
$$

and $r \sim s(r) \vee(s(r))$.

Putting (3.3) in (3.2) when $p=p *(r)$, we can obtain

$$
\mathrm{b} *(\mathrm{r}) \sim \mathrm{p} *(\mathrm{r})[1+o(1)] \sim \mathrm{s}(\mathrm{r})
$$

and so $b *(r) \sim \frac{r}{V(s(r))}$ for $r>0$. 
By the non-decreasing property of $\nu$ and $s$, there exists a continuous function $\psi(r)$, non-decreasing with $\psi(0)>0$ such that $b *(r) \sim \frac{r}{\psi(r)}$ for $r>0$.

Now let $\xi=\sup \left\{\left(\frac{M(j)}{M_{(j)}^{*}}\right)^{1 /|(j)|}\right\}<\infty$ and $n=\frac{1}{\xi}$.

We have $\lambda *(n r) \leq \lambda *(r)$ by Theorem 2 of [8] while $M_{(j)} \geq M_{(j)}^{*}$ implies $\lambda(r) \leq \lambda *(r)$. On the other hand, $\frac{M(j)}{\eta|(j)|}=M_{(j)}^{*} \xi^{|(j)|} \geq M_{(j)} \forall(j)$ implies $\lambda *(n r) \leq \lambda(r)$ by (3.1). Now, $M_{(j)}^{*}$ being quasi-analytic, the integral $\int_{1}^{\infty} \frac{b *(r)}{r^{2}} d r$ diverges and, by the above inequalities, implies the divergence of $\int_{1}^{\infty} \frac{b(r)}{r^{2}} d r$. If $f(t)=\boldsymbol{O}\left(e^{-|t| / \psi(|t|)}\right)$, then a fortiori $f(t)=\boldsymbol{O}(1 / \lambda(|t|))$ and by Theorem 1.3 , F has $(-\infty, \infty)$ as its spectrum.

The second part of the theorem follows from a result by E. Lukacs ([3, Theorem 3) combined with the regularity of $\mathrm{C}\left\{\mathrm{M}_{(j)}\right\}$. The proof, based on classical results of Khintchine, the inversion formula, and Parseval's Theorem, follows closely Lukacs (see [3]).

ACKNOWLEDGEMENTS. Research partia11y supported by NSERC (Canada) grant A9249. The author wishes to thank the referee for many helpful suggestions.

\section{REFERENCES}

1. LEVINSON, N. A Theorem Relating Non-Vanishing and Analytic Functions, J. Math. Phys. 16 (1938), 185-190.

2. KAWATA, T. Fourier Analysis in Probability Theory, Academic Press, New York and London, 1972 .

3. LUKACS, E. Analytical methods in Probability Theory Lecture Notes in Math, Vol. 31, (1967) Springer-Verlag, Berlin and New York.

4. KATZNELSON, Y. and MANDELBROJT, S. Fonctions entières et le problème de Gelfand et Silov, J. Analyse Math. 15 (1965), 263-279.

5. MANDELBROJT, S. Séries adhérentes, régularisation des suites, applications, Gauthier-Villars, Paris, 1952.

6. PHAM-GIA, T. Density of non quasi-analytic classes of functions, Canad. Math. Bu11. 20 (1977), 467-469.

7. LELONG, P. Sur une propriété de quasi-analyticité des fonctions de plusieurs variables, Comptes rendus Ac. Sc. Paris 232 (1951), 1178-1180.

8. LELONG, P. Extension d'un théorème de Carleman, Ann. Inst. Fourier, Grenoble 12. $(1962), 627-641$.

9. RUDIN, W. Real and Complex Analysis, McGraw-Hil1, New York, 1966.

10. GNEDENKO, B.V. Sur les fonctions caractéristiques, Bull. Univ. d'Etat à Moscou (Série Internationale) 1 (1973), 1-3. 
11. HIRSCHMAN, Jr., I.I. On the distribution of the zeros of functions belonging to certain quasi-analytic classes, Amer. J. Math. 72 (1950), 396-406. 


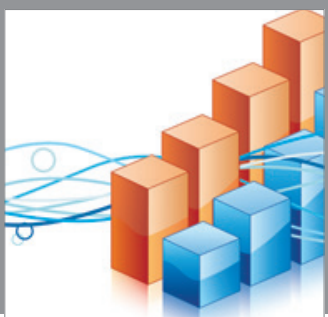

Advances in

Operations Research

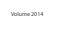

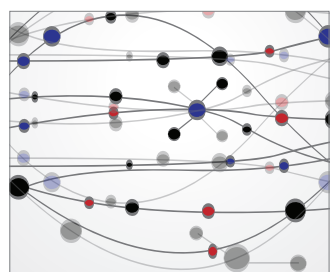

\section{The Scientific} World Journal
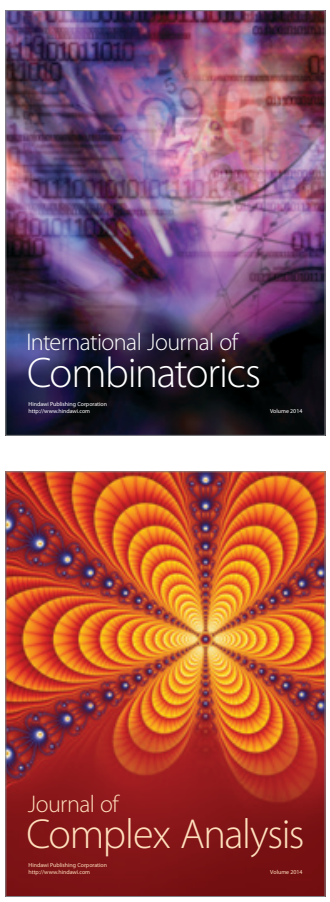

International Journal of

Mathematics and

Mathematical

Sciences
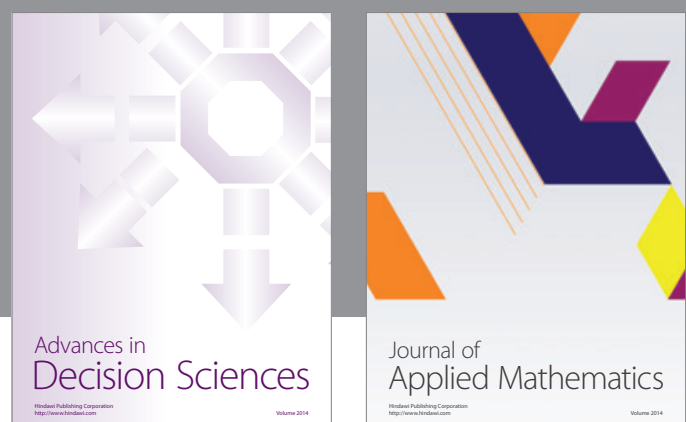

Journal of

Applied Mathematics
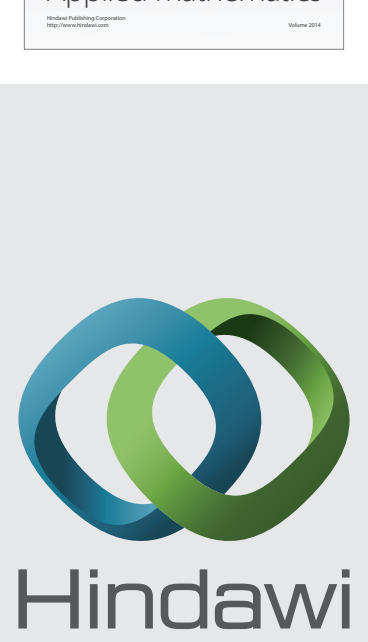

Submit your manuscripts at http://www.hindawi.com
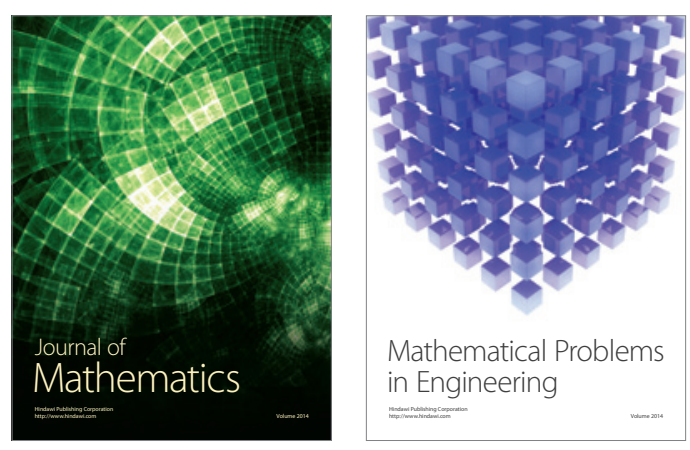

Mathematical Problems in Engineering
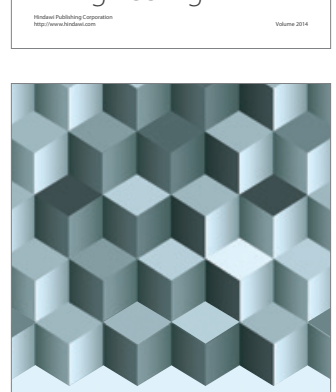

Journal of

Function Spaces
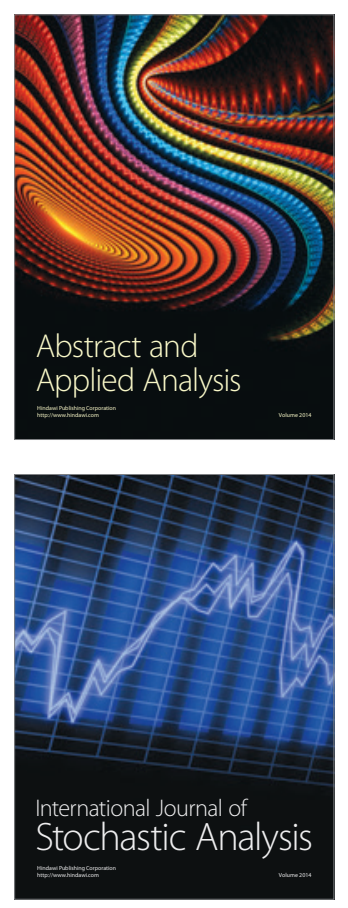

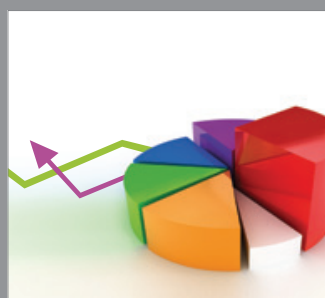

ournal of

Probability and Statistics

Promensencen
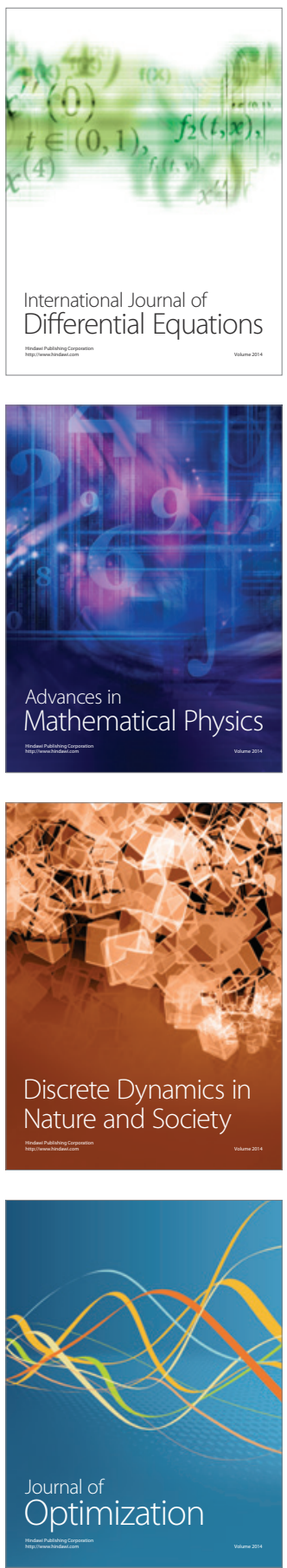